\title{
Probing Non-Abelian Statistics with Quasiparticle Interferometry
}

\author{
Parsa Bonderson, ${ }^{1}$ Kirill Shtengel, ${ }^{2,1}$ and J. K. Slingerland ${ }^{3}$ \\ ${ }^{1}$ California Institute of Technology, Pasadena, California 91125, USA \\ ${ }^{2}$ Department of Physics, University of California, Riverside, California 92521, USA \\ ${ }^{3}$ Microsoft Research, Station Q, Kohn Hall, University of California, Santa Barbara, California 93106, USA
}

(Received 28 January 2006; published 5 July 2006)

\begin{abstract}
We examine interferometric experiments in systems that exhibit non-Abelian braiding statistics, expressing outcomes in terms of the modular $S$-matrix. In particular, this result applies to fractional quantum Hall interferometry, and we give a detailed treatment of the Read-Rezayi states, providing explicit predictions for the recently observed $\nu=12 / 5$ plateau.
\end{abstract}

DOI: 10.1103/PhysRevLett.97.016401

Quantum systems in two spatial dimensions allow for exotic exchange statistics, characterized by a unitary representation of the braid group [1,2]. This representation may be non-Abelian, acting on a multidimensional internal Hilbert space [3,4]. So far, experimental evidence for the existence of such anyonic statistics has only recently been found in the (Abelian) Laughlin states of fractional quantum hall (FQH) systems [5]. However, the prospect of nonAbelian statistics is far more exciting, especially in light of its potential application in topologically fault-tolerant quantum computation [6,7]. There are currently several observed FQH states, at filling fractions $\nu=5 / 2,7 / 2$, $12 / 5$ [8] (and possibly $\nu=3 / 8,19 / 8$ ), that are expected to possess non-Abelian statistics. Numerical studies $[9,10]$ suggest that the $\nu=5 / 2,12 / 5$ states should be described, respectively, by the Moore-Read (MR) state [11] and the $k=3, M=1$ Read-Rezayi (RR) state [10]. Clearly, as experimental capabilities progress, it becomes increasingly important to understand how to probe and correctly identify the braiding statistics of quasiparticles. In this Letter, we explain how, for any system described by a topological quantum field theory (TQFT) in the infrared limit (e.g., FQH systems), knowledge of modular $S$-matrices may be used to extract this information from interferometry experiments or, inversely, to predict the outcomes of such experiments. As an example relevant to current experimental interests, we obtain explicit results for the RR states.

The topological properties of 2D quantum systems with an energy gap can be described using TQFTs (or "modular tensor categories" in mathematicians' terminology, see, e.g., [12-14]), often abstracted from conformal field theories (CFTs, see [15] and references therein). Such anyon models are defined by (i) a finite set $\mathcal{C}$ of particle types or "anyonic charges," (ii) fusion rules specifying how these particle types may combine or split, and (iii) braiding rules dictating the behavior under exchange of two particles (all subject to certain consistency conditions). The "vacuum" charge is given the label $\mathbb{1}$. The antiparticle or "charge conjugate" of a particle type $a$ is denoted $\bar{a}$, and is the unique charge that can fuse with $a$ to give $\mathbb{1}$. Fusion of particle types generalizes the addition of charges or angu-
PACS numbers: 71.10.Pm, 11.25.Hf, 73.43.Fj, 73.43.Jn

lar momenta, and the (commutative and associative) fusion rules are specified as $a \times b=\sum_{c \in \mathcal{C}} N_{a b}^{c} c$, where the integer $N_{a b}^{c}$ is the dimension of the Hilbert space of particles of type $a$ and $b$ restricted to have total anyonic charge $c$. Fusion and braiding can be represented diagrammatically by oriented, labeled particle worldlines, and are unaffected by smooth deformations in which the lines do not intersect. Charge conjugation is represented by reversal of worldline orientation. We will refer to only one braiding relation, known as the modular $S$-matrix, defined by the diagram

$$
S_{a b}=\frac{1}{D} \overbrace{b} \text {. }
$$

Here $D=\sqrt{\sum_{a} d_{a}^{2}}=1 / S_{\mathbb{1} \mathbb{1}}$ is the total quantum dimension, where $d_{a}$, the quantum dimension of particle type $a$, is the value of a single loop of that type,

$$
d_{a}=\bigcirc a=D S_{\mathbb{1} a} .
$$

Some useful properties of the $S$-matrix are

$$
S_{a b}=S_{b a}={\overline{\left(S^{-1}\right)_{a b}}}=\bar{S}_{\bar{a} b} .
$$

The importance of the $S$-matrix becomes clear when one envisions interferometry experiments for these systems in which a particle has two possible paths that it may take around another particle, the two paths combining to form a closed loop. This is typical of Mach-Zender, two-slit, FQH two-point-contact, etc., experiments [16-22]. In such experiments, an interference term arises that can be written as

$$
\left\langle\Psi_{a b}\left|U_{1}^{-1} U_{2}\right| \Psi_{a b}\right\rangle=e^{i \alpha_{a b}}\left\langle\Psi_{a b}|\mathbb{M}| \Psi_{a b}\right\rangle=e^{i \alpha_{a b}} M_{a b},
$$

where $\left|\Psi_{a b}\right\rangle$ is the initial state of particles $a$ and $b$, and $U_{1}$, $U_{2}$ are the unitary evolution operators for the particle $a$ traveling around the particle $b$ via the two respective paths. It has been rewritten in terms of the monodromy operator $\mathbb{M}$ that contains only the contribution from adiabatically transporting particle $a$ around particle $b$ (i.e., braiding), and a phase $e^{i \alpha_{a b}}$ that absorbs all other contributions (i.e., it 
contains the free particle dynamics and the AharonovBohm phase from a background magnetic flux). For Abelian particles, $\left|M_{a b}\right|=1$, but with non-Abelian statistics, $\left|M_{a b}\right|$ may be less than 1 and can be calculated by TQFT methods. The braiding term is diagrammatically represented by winding the worldline of particle $a$ around that of particle $b$, taking the standard closure (where each worldline is closed back on itself without additional braiding) and dividing by the quantum dimension of the two particle types [23]. Thus, the resulting monodromy matrix element can be written entirely in terms of the $S$-matrix:

$$
M_{a b}=\frac{1}{d_{a} d_{b}} \bigcirc=\frac{S_{a b} S_{\mathbb{1}}}{S_{\mathbb{1} a} S_{\mathbb{1} b}} .
$$

This result is particularly nice because the $S$-matrix is typically more readily computable than the complete set of braiding or fusion rules, and, in fact, has already been computed for most physically relevant theories. In particular, this applies to the class of theories described by CFTs generated as products and cosets of Wess-Zumino-Witten theories, which includes all proposed non-Abelian FQH states (see [24]). In the case of the product of two CFTs or TQFTs, the particle types are denoted by pairs of particle labels, one from each theory, and the $S$-matrix of the product theory is the tensor product of the $S$-matrices of the parent theories. In the $G_{k} / H_{l}$ coset theory, the new labels are also formed as pairs of labels from the parent $G_{k}$ and $H_{l}$ theories, but now there are branching rules which restrict the allowed pairings. Also, different pairs of labels may sometimes turn out to represent the same particle type, a phenomenon described by "field identifications." This means that there will be only one row and column in the $S$-matrix for any set of identified labels. Despite these complications, the coset's $S$-matrix elements are described by the simple formula [25]

$$
S_{(a, p)(b, q)}^{(G / H)}=c(G, H, k, l) S_{a b}^{(G)} \bar{S}_{p q}^{(H)},
$$

where $a, b$ and $p, q$ are, respectively, labels of the $G_{k}$ and $H_{l}$ theories, and $c(G, H, k, l)$ is an overall normalization constant that enforces unitarity of the $S$-matrix [but is irrelevant in Eq. (5)].

We now focus specifically on $\mathrm{FQH}$ systems, because they represent the most likely physical candidates for finding non-Abelian statistics. We consider the experiment originally proposed in [17] for measuring braiding statistics in the Abelian FQH states, which was later adopted for the non-Abelian case in [18] and addressed again in the context of the $\nu=5 / 2$ state in [21,22]. A somewhat similar experiment has recently been implemented to probe the Abelian $\nu=1 / 3$ state [5]. The experimental setup is a two point-contact interferometer composed of a quantum Hall bar with two front gates on either side of an antidot (see Fig. 1). Biasing the front gates, one may create constrictions in the Hall bar, adjusting the tunneling amplitudes $t_{1}$ and $t_{2}$. Tunneling between the opposite edge currents leads to a deviation of $\sigma_{x y}$ from its quantized value, or equivalently to the appearance of $\sigma_{x x}$. By measuring $\sigma_{x x}$ one effectively measures the interference between the two tunneling paths around the antidot. The tunneling amplitudes $t_{1}$ and $t_{2}$ must be kept small, to ensure that the tunneling current is completely due to quasiholes rather than higher charge composites [26] and to allow us to restrict our attention to the lowest order winding term.

In order to be able to influence the resulting interference pattern, we envision several experimentally variable parameters: (i) the central gate voltage allowing one to control the number $n$ of quasiholes on the antidot, (ii) the perpendicular magnetic field $B$, (iii) the back gate voltage controlling the uniform electron density, and (iv) the side gate that can be used to modify the shape of the edge inside the interferometric loop [21]. The intention is to be able to separately affect the Abelian Aharonov-Bohm phase and the number of quasiholes on the antidot; from this point of view having all these controls is redundant, but may be beneficial for experimental success.

The longitudinal conductivity is proportional to the probability that current entering the bottom edge will leave through the top edge, which to lowest order is

$$
\begin{aligned}
\sigma_{x x} & \propto\left|t_{1}\right|^{2}+\left|t_{2}\right|^{2}+2 \operatorname{Re}\left\{t_{1}^{*} t_{2}\left\langle\Psi_{a b}\left|U_{1}^{-1} U_{2}\right| \Psi_{a b}\right\rangle\right\} \\
& =\left|t_{1}\right|^{2}+\left|t_{2}\right|^{2}+2\left|t_{1} t_{2}\right|\left|M_{a b}\right| \cos \left(\beta+\theta_{a b}\right) .
\end{aligned}
$$

In this equation $\beta=\alpha_{a b}+\arg \left(t_{2} / t_{1}\right)$ can be varied by changing $B$ (keeping the quasihole number fixed), the relative tunneling phase, and/or the edge shape around the central region. We have written $M_{a b}=\left|M_{a b}\right| e^{i \theta_{a b}}$, and will be interested in the elements where the particle $a$ which tunnels carries the anyonic charge of a quasihole, while the "particle" $b$ is a composite of $n$ quasiholes on the antidot, carrying an anyonic charge allowed by fusion [27].

We will now apply this formalism to the RR states and, in particular, look more closely at the $k=3, M=1$ case, which, by a particle-hole transformation (which generally inverts the statistics and has the effect of conjugating the $S$-matrix), is the expected description of $\nu=12 / 5$. The

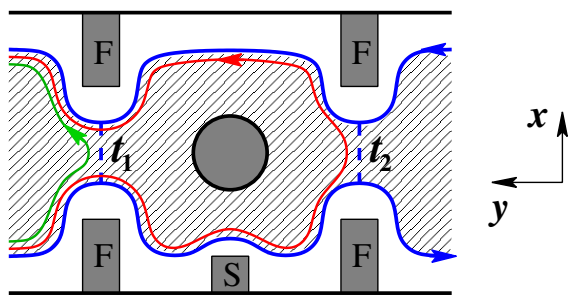

FIG. 1 (color online). A two point-contact interferometer for measuring braiding statistics. The hatched region contains a $\mathrm{FQH}$ liquid. The front gates (F) are used to bring the opposite edge currents (indicated by arrows) close to each other to form two tunneling junctions. Applying voltage to the central gate creates an antidot in the middle and controls the number $n$ of quasiholes contained there. A side gate $(S)$ can be used to change the shape and path length in the interferometer. 
anyon theory for RR states at $\nu=k /(k M+2)$ can be described as $\mathrm{RR}_{k, M}=\mathrm{U}(1)_{k, M} \times \mathrm{Pf}_{k}$, where $\mathrm{U}(1)_{k, M}$ is due to the electric charge and $\mathrm{Pf}_{k}$ represents the $\mathbb{Z}_{k}$-parafermion theory [28]. The $\mathrm{U}(1)_{k, M}$ part of this theory is a simple Abelian contribution, essentially labeled by integral multiples of the charge/flux unit $\left(\frac{e}{k M+2}, \frac{2 \pi}{k e}\right)$, where $-e$ is the electron charge and $\frac{2 \pi}{e}=\Phi_{0}$ is the magnetic flux quantum (in units $\hbar=c=1$ ). The fusion rules for these labels are just the addition of charge/flux and the $S$-matrix is $S_{n_{a} n_{b}}=e^{i n_{a} n_{b} 2 \pi / k(k M+2)} / \sqrt{k(k M+2)}$.

The $\mathrm{Pf}_{k}$ part of this theory requires more explanation (for a discussion of its braiding, see [29]). Essentially, we use that the theory is equivalent to the coset $\mathrm{SU}(2)_{k} / \mathrm{U}(1)_{k}$. As a consequence, the $\mathbb{Z}_{k}$-parafermion sector's anyonic charge can be labeled by the corresponding CFT fields $\Phi_{\lambda}^{\Lambda}$, where $\Lambda \in\{0,1, \ldots, k\}$ and $\lambda \in \mathbb{Z}$, subject to the identifications $\Phi_{\lambda}^{\Lambda}=\Phi_{\lambda+2 k}^{\Lambda}=\Phi_{\lambda-k}^{k-\Lambda}$ and the restriction $\Lambda+\lambda \equiv 0(\bmod 2)$, giving a total of $\frac{1}{2} k(k+1)$ fields. The fusion rules for this sector are (as for the CFT fields)

$$
\Phi_{\lambda_{a}}^{\Lambda_{a}} \times \Phi_{\lambda_{b}}^{\Lambda_{b}}=\sum_{\Lambda=\left|\Lambda_{a}-\Lambda_{b}\right|}^{\min \left\{\Lambda_{a}+\Lambda_{b}, 2 k-\Lambda_{a}-\Lambda_{b}\right\}} \Phi_{\lambda_{a}+\lambda_{b}}^{\Lambda} .
$$

Their quantum dimensions are

$$
d_{\Phi_{\lambda}^{\Lambda}}=\sin \left(\frac{(\Lambda+1) \pi}{k+2}\right) / \sin \left(\frac{\pi}{k+2}\right) .
$$

Special fields in this theory are the vacuum $\mathbb{1} \equiv \Phi_{0}^{0}$, the $\mathbb{Z}_{k}$-parafermions $\psi_{l} \equiv \Phi_{2 l}^{0}$, the primary fields $\sigma_{l} \equiv \Phi_{l}^{l}$, and the $\mathbb{Z}_{k}$-neutral excitations $\varepsilon_{j} \equiv \Phi_{0}^{2 j}$, where $l=$ $1, \ldots, k-1$ and $j=1, \ldots,\lfloor(k-1) / 2\rfloor$. From (6) we find that the $S$-matrix for $\operatorname{Pf}_{k}$ is

$$
S_{\Phi_{\lambda_{a}}^{\Lambda_{a}} \Phi_{\lambda_{b}}^{\Lambda_{b}}}=\frac{\sin \left(\frac{\left(\Lambda_{a}+1\right)\left(\Lambda_{b}+1\right) \pi}{k+2}\right)}{D \sin \left(\frac{\pi}{k+2}\right)} e^{-i \lambda_{a} \lambda_{b} \pi / k} .
$$

The $\mathrm{U}(1)_{k, M}$ and $\mathrm{Pf}_{k}$ sectors combine so that the anyonic charges in the $\mathrm{RR}_{k, M}$ theory are (defining a shorthand) $\hat{\Lambda}_{n} \equiv\left(\frac{n e}{k M+2}, \frac{n 2 \pi}{k e}, \Phi_{n}^{\Lambda_{n}}\right)$, where we have $n \in \mathbb{Z}$ and $\Lambda_{n} \in$ $\{0,1, \ldots, k\}$ such that $\Lambda_{n}+n \equiv 0(\bmod 2)$. Quasiholes carry charge $\hat{1}_{1}=\left(\frac{e}{k M+2}, \frac{2 \pi}{k e}, \sigma_{1}\right)$. The $S$-matrix for RR anyons is obtained by multiplying the $S$-matrix elements of the two sectors for anyons $a$ and $b$, and renormalizing by some overall constant $c$ (which we will not need explicitly):

$$
S_{a b}=c \sin \left(\frac{\left(\Lambda_{n_{a}}+1\right)\left(\Lambda_{n_{b}}+1\right) \pi}{k+2}\right) e^{-i n_{a} n_{b} M \pi /(k M+2)} .
$$

Since the tunneling current is dominated by quasiholes [30], we need only the monodromy matrix elements

$$
M_{\hat{1}_{1}, \hat{\Lambda}_{n}}=\frac{\cos \left(\frac{\left(\Lambda_{n}+1\right) \pi}{k+2}\right)}{\cos \left(\frac{\pi}{k+2}\right)} e^{-i n M \pi /(k M+2)} .
$$

We note that $\mathrm{RR}_{2,1}$ is the MR state, and we can easily check that this exactly matches the results of [18,22].

We now turn to the $\mathrm{RR}_{3,1}$ theory for $\nu=12 / 5$. The $\mathrm{Pf}_{3}$ theory has six fields: $\mathbb{1}, \psi_{1}, \psi_{2}$, which have quantum dimension 1, and $\sigma_{1}, \sigma_{2}, \varepsilon$, which have quantum dimen$\operatorname{sion} \phi=2 \cos \frac{\pi}{5}=\frac{1+\sqrt{5}}{2}$ (the golden ratio). The total quantum dimension is $D=\sqrt{3(\phi+2)}$ and the $S$-matrix is

$$
S=\frac{1}{D}\left[\begin{array}{cccccc}
1 & 1 & 1 & \phi & \phi & \phi \\
1 & \omega & \bar{\omega} & \phi \bar{\omega} & \phi \omega & \phi \\
1 & \bar{\omega} & \omega & \phi \omega & \phi \bar{\omega} & \phi \\
\phi & \phi \bar{\omega} & \phi \omega & -\omega & -\bar{\omega} & -1 \\
\phi & \phi \omega & \phi \bar{\omega} & -\bar{\omega} & -\omega & -1 \\
\phi & \phi & \phi & -1 & -1 & -1
\end{array}\right]
$$

where $\omega=e^{i 2 \pi / 3}$, and the columns and rows are in the order: $\mathbb{1}, \psi_{1}, \psi_{2}, \sigma_{1}, \sigma_{2}, \varepsilon$. Quasiholes in the $\mathrm{RR}_{3,1}$ theory have anyonic charge $\left(\frac{e}{5}, \frac{2 \pi}{3 e}, \sigma_{1}\right)$. It is useful to consider a Bratteli diagram (which has periodicity 6 in $n$ ) to keep track of the allowed $\mathrm{Pf}_{3}$ charge for a corresponding value of $n$ :

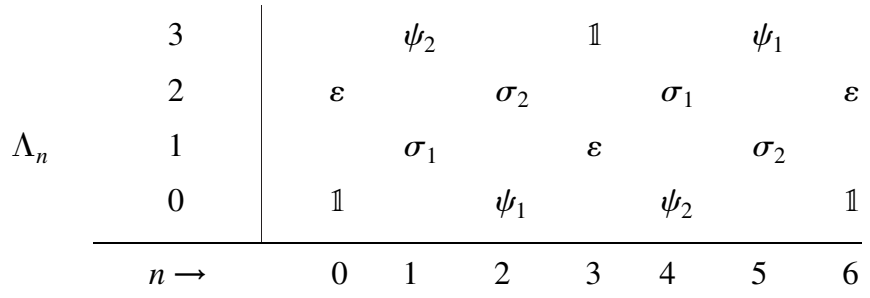

The longitudinal conductivity in the interferometry experiment will be

$$
\sigma_{x x} \propto\left|t_{1}\right|^{2}+\left|t_{2}\right|^{2}+2\left|t_{1} t_{2}\right|\left(-\phi^{-2}\right)^{N_{\phi}} \cos \left(\beta+n \frac{4 \pi}{5}\right),
$$

where $N_{\phi}=1$ if the $n$ quasihole composite on the antidot has $\mathrm{Pf}_{3}$ charge with quantum dimension $\phi$ (i.e., $\sigma_{1}, \sigma_{2}$, or $\varepsilon$ ) and $N_{\phi}=0$ if the composite has quantum dimension 1 (i.e., $\mathbb{1}, \psi_{1}$, or $\psi_{2}$ ). Thus, depending on the total $\mathrm{Pf}_{3}$ charge on the antidot, one of two possible conductivity values will be observed. The $\mathrm{Pf}_{3}$ charge may then be determined by varying $\beta$ (using the side gate) to measure the interference fringe amplitude, which is suppressed by a factor of $\phi^{-2} \approx$ 0.38 when $N_{\phi}=1$. This behavior indicates the presence of non-Abelian statistics, and distinguishes this state from other proposals for the same filling fraction (e.g. composite fermions). To describe $\nu=12 / 5$, we apply a particle-hole transformation to $\mathrm{RR}_{3,1}$, replacing $S$ with $\bar{S}$ (hence, $M$ with $\bar{M})$, which changes the sign in front of $n$ in Eq. (13). In more general scenarios where composites of quasiholes or quasielectrons may be used instead of the single tunneling quasiholes, identical behavior (up to the phase) will be exhibited by quantum dimension $\phi$ composites, while quantum dimension 1 composites exhibit a single unsuppressed interference pattern at any $n$.

Despite the relatively simple nature of these interferometry experiments, they provide a surprisingly large amount of information about the system being probed. This is because the experiments essentially measure the $S$-matrix of the TQFT that describes the system. The $S$-matrix fully 
determines the fusion rules through the Verlinde formula [31]: $N_{a b}^{c}=\sum_{x \in \mathcal{C}} S_{a x} S_{b x} S_{\bar{c} x} / S_{11 x}$. Additionally, a theorem known as "Ocneanu rigidity" states that, given a set of fusion rules, there are only finitely many corresponding TQFTs with these rules [32]. In other words, knowledge of the $S$-matrix is sufficient to pin down the topological order of the state to a finite number of possibilities. Clearly, it may be difficult to measure all elements of the $S$-matrix by the methods described here. It appears particularly challenging to invoke tunneling of anyonic charges different from that of the quasihole. Still, the $S$-matrix has many special properties and so even a partial measurement of fairly low accuracy may be sufficient to determine it. In addition to Eq. (3), any $S$-matrix must satisfy a set of constraints coming from the Verlinde formula and the fact that the fusion coefficients are integers. Also, the first row of the $S$-matrix must be real and positive, because of its relation to the quantum dimensions (and in fact, all elements are numbers with special algebraic properties). Finally, given an $S$-matrix, there must be a diagonal matrix $T$ which together with $S$ generates a representation of the modular group $\operatorname{SL}(2, \mathbb{Z})$, implying that $(S T)^{3}=S^{2}$. For any fixed number of particle types, only finitely many different $S$-matrices are known (and it is conjectured that only finitely many exist). Hence, once some the $S$-matrix elements and the number of different charges are known from measurements, one may look at the finite list of known $S$-matrices and hope to identify one that matches. In conclusion, for any two-dimensional system, interference experiments as described here can in principle determine the fusion rules and even a finite set of TQFTs, one of which will fully describe the topological order.

The authors would like to thank E. Ardonne, S. B. Chung, P. Fendley, A. Kitaev, C. Nayak, J. Preskill, and Z. Wang for many illuminating discussions. This work was supported in part by the NSF under Grant No. EIA0086038. P. B. and K. S. would like to acknowledge the hospitality of Microsoft Project Q and KITP. K. S. is also grateful for the hospitality of the IQI.

[1] J. M. Leinaas and J. Myrheim, Nuovo Cimento B 37, 1 (1977).

[2] F. Wilczek, Phys. Rev. Lett. 49, 957 (1982).

[3] G. A. Goldin, R. Menikoff, and D. H. Sharp, Phys. Rev. Lett. 54, 603 (1985).

[4] J. Fröhlich, in Non-Perturbative Quantum Field Theory, edited by G. 't Hooft (Plenum, New York, 1988).

[5] F. E. Camino, W. Zhou, and V. J. Goldman, Phys. Rev. B 72, 075342 (2005); Phys. Rev. Lett. 95, 246802 (2005).

[6] A. Y. Kitaev, Ann. Phys. (N.Y.) 303, 2 (2003).

[7] M.H. Freedman, M. J. Larsen, and Z. Wang, Commun. Math. Phys. 227, 605 (2002).

[8] R. Willett et al., Phys. Rev. Lett. 59, 1776 (1987); W. Pan et al., ibid. 83, 3530 (1999); J. S. Xia et al., ibid. 93, 176809 (2004).
[9] R. H. Morf, Phys. Rev. Lett. 80, 1505 (1998); E. H. Rezayi and F. D. M. Haldane, ibid. 84, 4685 (2000).

[10] N. Read and E. Rezayi, Phys. Rev. B 59, 8084 (1999).

[11] G. Moore and N. Read, Nucl. Phys. B360, 362 (1991).

[12] J. Preskill, lecture notes, http://www.theory.caltech.edu/ preskill/ph219/topological.ps (2004).

[13] A. Kitaev, Ann. Phys. (N.Y.) 321, 2 (2006).

[14] V.G. Turaev, Quantum Invariants of Knots and 3-Manifolds (Walter de Gruyter, Berlin, 1994); C. Kassel, Quantum Groups (Springer, New York, 1995).

[15] F. Di Francesco, P. Mathieu, and D. Sénéchal, Conformal Field Theory (Springer, New York, 1997).

[16] E. Verlinde, in Proceedings of the International Colloquium on Modern Quantum Field Theory, Bombay, India, 1990 (World Scientific, Singapore, 1991); H.-K. Lo and J. Preskill, Phys. Rev. D 48, 4821 (1993); F. A. Bais et al., Phys. Lett. B 280, 63 (1992); Nucl. Phys. B393, 547 (1993).

[17] C. de C. Chamon et al., Phys. Rev. B 55, 2331 (1997).

[18] E. Fradkin et al., Nucl. Phys. B516, 704 (1998).

[19] B. J. Overbosch and F. A. Bais, Phys. Rev. A 64, 062107 (2001).

[20] S. Das Sarma, M. Freedman, and C. Nayak, Phys. Rev. Lett. 94, 166802 (2005).

[21] A. Stern and B. I. Halperin, Phys. Rev. Lett. 96, 016802 (2006).

[22] P. Bonderson, A. Kitaev, and K. Shtengel, Phys. Rev. Lett. 96, 016803 (2006).

[23] This result is derived for initial states of two uncorrelated particles. Other initial states and correlations may require more involved arguments along the lines set out in [19].

[24] J. Fröhlich et al., J. Stat. Phys. 103, 527 (2001); X. G. Wen, Phys. Rev. Lett. 66, 802 (1991); E. Ardonne and K. Schoutens, ibid. 82, 5096 (1999); E. Ardonne et al., Phys. Rev. B 65, 041305(R) (2002).

[25] D. Gepner, Phys. Lett. B 222, 207 (1989).

[26] In the weak tunneling regime, the tunneling current $I \propto$ $V^{2 s-1}$, where $s$ is the scaling dimension or topological spin of the corresponding fields [33]. It follows that the dominant contribution is from the field with lowest scaling dimension, which in $\mathrm{FQH}$ systems is the quasihole.

[27] We expect the states of the antidot with the same electric charge but different topological spins to be nondegenerate (with energy difference scaling as $L^{-1}$ for an antidot of size $L$ ) and to have different charge distributions, ruling out their superposition over extended time.

[28] A. B. Zamolodchikov and V. Fateev, Sov. Phys. JETP 62, 215 (1985); D. Gepner and Z. Qiu, Nucl. Phys. B285, 423 (1987).

[29] J. K. Slingerland and F. A. Bais, Nucl. Phys. B612, 229 (2001).

[30] As previously noted, the particle with lowest topological spin dominates tunneling. For $\mathrm{RR}_{k, M}$ particles with charge $\hat{\Lambda}_{n}$, the topological spin is given by $s_{\hat{\Lambda}_{n}}=\frac{n^{2}}{2 k(k M+2)}+$ $\frac{\Lambda(\Lambda+2)}{4(k+2)}-\frac{\lambda^{2}}{4 k}$, where the field identifications must be used to write $\Phi_{n}^{\Lambda_{n}}=\Phi_{\lambda}^{\Lambda}$ such that $-\Lambda \leq \lambda \leq \Lambda$ in order to apply this formula. All other particles have greater spin than the quasihole value, $s_{\hat{1}_{1}}=\frac{k M-M+3}{2(k+2)(k M+2)}$.

[31] E. Verlinde, Nucl. Phys. B300, 360 (1988).

[32] P. Etingof et al., Ann. Math. 162, 581 (2005).

[33] X. G. Wen, Int. J. Mod. Phys. B 6, 1711 (1992). 